\title{
Pressure and non-linear quark number susceptibilities in QCD
}

\author{
R. V. Gavai ${ }^{\mathrm{a}}$ and Sourendu Gupta ${ }^{\mathrm{a} *}$. \\ ${ }^{a}$ Dept. of Theoretical Physics, Tata Institute of Fundamental Research, Homi Bhabha Road, Mumbai \\ 400005, India
}

We report the first measurements of sixth order non-linear quark number susceptibilities in continuum QCD. This extends our earlier computation of the continuum limit [1] of non-linear quark number susceptibilities and the pressure in QCD for $m / T_{c}=0.1$. For $T / T_{c} \geq 1.5$, no phase transitions are observable for $\mu<30 T$. Systematics of off-diagonal susceptibilities are reported. The equation of state in the high temperature phase of the QCD plasma is constructed.

The derivatives of the pressure with respect to the quark chemical potentials (one for each flavour)-

$\chi_{f g h \cdots}^{(l)}=\frac{\partial^{l} P}{\partial \mu_{f} \partial \mu_{g} \partial \mu_{h} \cdots}$

are the quark number susceptibilities (QNS, for $l \geq 2)$ and the number densities $(n$, for $l=1)$. Thus, the quark number susceptibilities measured at zero chemical potential (where direct lattice computations are possible) allow reconstruction of the equation of state at finite chemical potential through the usual multivariate Taylor series expansion-

$\Delta P(T,\{\mu\})=\sum_{l ; f g h \ldots} \frac{1}{l !} \chi_{f g h \ldots}^{(l)} \mu_{f} \mu_{g} \mu_{h} \cdots$,

where $\Delta P(T,\{\mu\})=P(T,\{\mu\})-P(T,\{0\})$. The coefficients for odd $l$ vanish due to CP symmetry. The convergence of eq. (2) is spoilt by phase transitions; hence any bound on the radius of convergence limits the location of the nearest phase transition to the point at $\mu=0$. Due to finite lattice spacing ambiguities in $\chi_{f g h \ldots}^{(n)}$, and the rapid convergence of the Taylor expansion away from a phase transition, the best way to compute the continuum equation of state (EOS) is through the Taylor expansion [1]. We show later that, for $T>T_{c}$, the equation of state [213] is dominated by the $l=2$ term (here $T_{c}$ means the transition

\footnotetext{
${ }^{*}$ By whom the talk was presented
}

or crossover point for $\mu=0$ ). The QNS are also interesting in themselves [4.

We report measurements of the continuum limit of the susceptibilities upto order 6 in $\mathrm{QCD}$ using the quenched approximation for $T \geq 1.5 T_{c}$, where the difference between quenched and dynamical computations is expected to be of the order of $5-10 \%$. Near $T_{c}$, where the difference is large, we show results obtained at finite cutoff in dynamical two-flavour computations.

Off-diagonal susceptibilities need the computation of quantities such as $\operatorname{tr} A^{n}$ for matrices $A$. Independent sets of $N_{v} / n$ random complex Gaussian vectors, $\{r\},\{s\}$, etc., are used for the noisy evaluation such as $\operatorname{tr} A^{2}=\overline{\langle r|A| r\rangle\langle s|A| s\rangle}$. For the evaluation of $\varnothing_{11}$, where $A$ is anti-Hermitean, the result is negative: in the $N_{v} \rightarrow \infty$ limit, the histogram of noisy evaluations is skew, peaking at zero, with a tail to the left, and vanishing on the right. As shown in Figure 1 fairly large $N_{v}$ are needed to get a signal (we used $\operatorname{Im}\langle r|A| r\rangle$ for noise reduction) near $T_{c}$. For larger $T$ even $N_{v}=100$ does not give a signal [1]. The conjugate gradient stopping criterion is not a crucial parameter in this computation. Of the offdiagonal susceptibilities, only $\chi_{u d}$ and $\chi_{u u d d}$ were found to differ from zero significantly near $T_{c}$ (degenerate 3-flavour QNS such as $\chi_{u d s}$ and $\chi_{u d s s}$ also vanish within errors). The temperature dependence of these quantities is shown in Figure 2 The peaks in these quantities close to $T_{c}$ are crucial for building up the peaks in the pressure near the critical end point [3], since the diagonal 
susceptibilities go smoothly to zero near $T_{c}$.

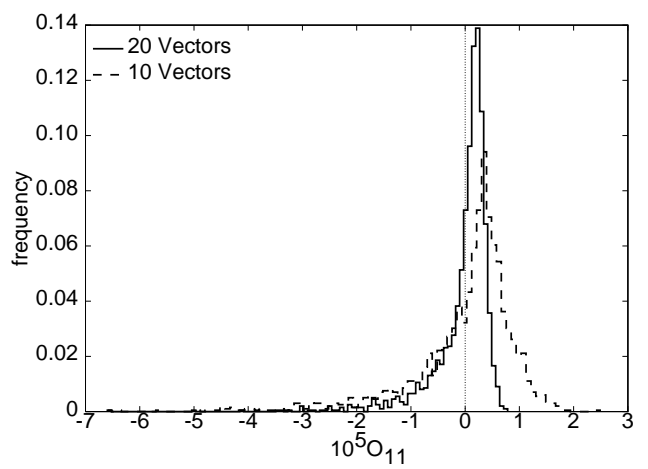

Figure 1. Normalised histogram of measurements of $\emptyset_{11}$ with different $N_{v}$ for dynamical $N_{f}=2$ computations at $1.05 T_{c}$ on a $4 \times 10^{3}$ lattice with $m / T_{c}=0.1$.
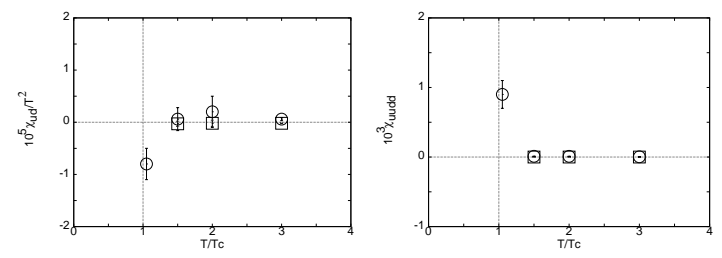

Figure 2. Temperature dependence of $\chi_{u d}$ (left panel) and $\chi_{\text {uudd }}$ (right panel). Boxes denote data for quenched QCD with $N_{t}=14$, and circles for dynamical $N_{f}=2 \mathrm{QCD}$ with $N_{t}=4$.

The 6th order diagonal QNS, $\chi_{\text {uuuuuu, can }}$ be reconstructed by standard methods from the sixth derivative of $Z$ -

$$
\begin{aligned}
& Z_{\text {ииииии }}=Z\left\langle\varnothing_{111111}+14 \varnothing_{11112}+20 \emptyset_{1113}\right. \\
& +45 \varnothing_{1122}+15 \varnothing_{114}+60 \varnothing_{123}+6 \varnothing_{15} \\
& \left.+15 \varnothing_{222}+15 \varnothing_{24}+10 \varnothing_{33}+\emptyset_{6}\right\rangle
\end{aligned}
$$

where the operators other than $\varnothing_{6}$ have been written out in [1]. In the notation of 1] this operator is given by

$$
\begin{aligned}
& \varnothing_{6}=-120(6 \cdot 1)+360(4 \cdot 1 \oplus 2) \\
& -210(2 \cdot 1 \oplus 2 \cdot 2)-60(1 \oplus 2 \oplus 1 \oplus 2) \\
& -120(3 \cdot 1 \oplus 3)+30(3 \cdot 2)+60(1 \oplus 2 \oplus 3) \\
& +60(1 \oplus 3 \oplus 2)+30(2 \cdot 1 \oplus 4)-10(2 \cdot 3) \\
& -15(2 \oplus 4)-6(1 \oplus 5)+(6)
\end{aligned}
$$

Results for the 6 th order susceptibilities are shown in Table 11 The large finite lattice size effects shown in Table 1 and in the other QNS 1 underline the need for taking the continuum limit in order to get reliable estimates of the location of the critical end point, where the Taylor expansion diverges due to the growth of the higher order susceptibilities.

Estimates of the radius of convergence of eq. (2) at the 4 th and 6 th orders are

$\frac{\mu_{*}^{(4)}}{T}=\sqrt{\frac{12 \chi_{u u} / T^{2}}{\chi_{u u u u}}}, \quad \frac{\mu_{*}^{(6)}}{T}=\sqrt{\frac{30 \chi_{u u u u}}{T^{2} \chi_{\text {uиuиuu }}}}$.

$\mu_{*}^{(4)} / T \approx 4.6$ in the continuum limit for $1.5 \leq$ $T / T_{c} \leq 31$, whereas $\mu_{*}^{(6)} / T \approx 28$ for $N_{t}=14$ and is possibly significantly larger in the continuum. For $T>T_{c}, \mu_{*}$ limit the phase boundary between the plasma and the appropriate coloursuperconducting phase. If the phase boundary is further off, or if, for $T<T_{c}$, there is quarkhadron continuity [5], then these two numbers are the value of $\mu / T$ at which the two terms being compared give equal contribution in the Taylor expansion. At SPS energies, where $\mu / T_{c}=0.45$ 6 , the 4 th order term gives a $5 \%$ correction to $\Delta P / T^{4}$, and the 6 th order term is totally negligible for $T / T_{c} \geq 1.5$. At the RHIC, where $\mu / T_{c}=0.15$, the leading term contributes more than $99 \%$ of the total.

The relation between $\Delta P(T,\{\mu\}) / T^{4}$ and the quark number density $n(T,\{\mu\}) / T^{3}$ is the equation of state 3. Since $n$ is the first derivative of the pressure, the EOS is completely determined once the susceptibilities are known. As shown in Figure 3, the difference between the leading term involving $\chi_{u u}$, and the next term, becomes important only when $n / T^{3} \approx 1$, i.e., for $\mu \approx T$. 


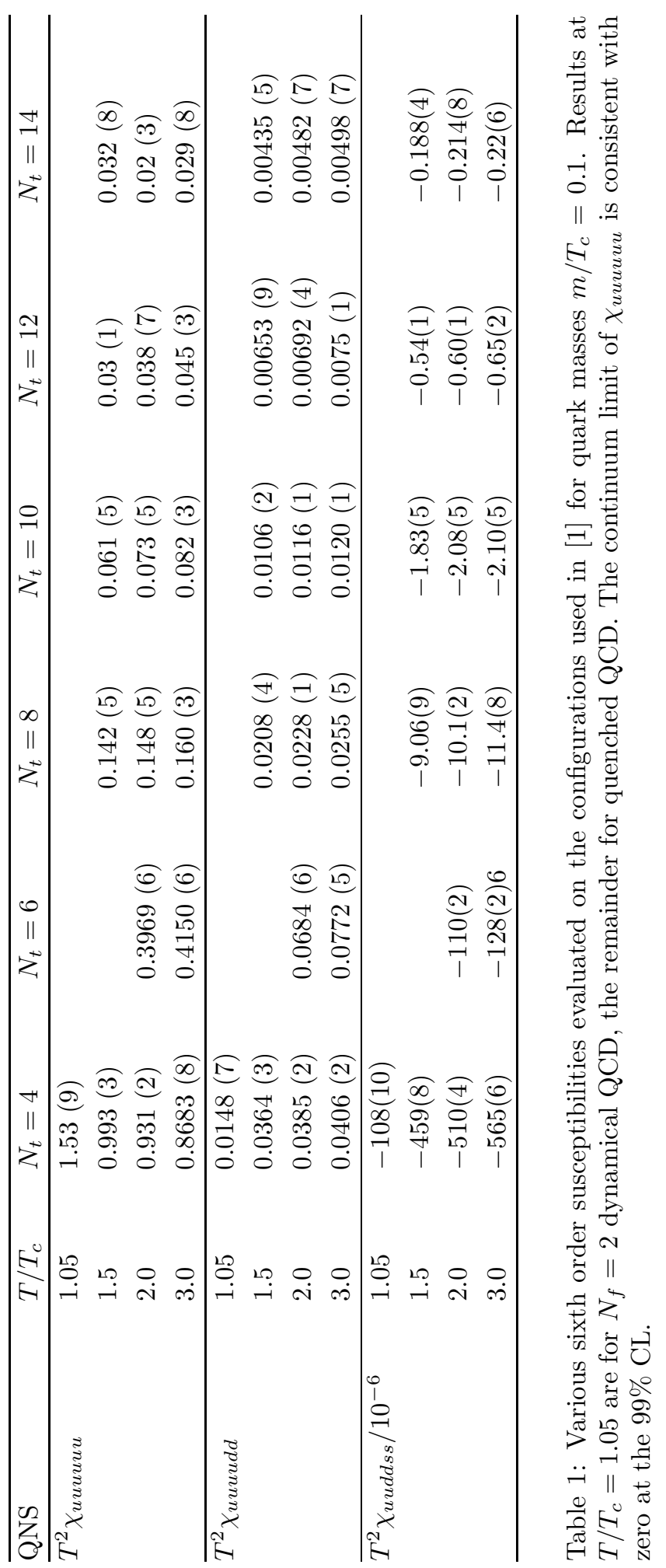

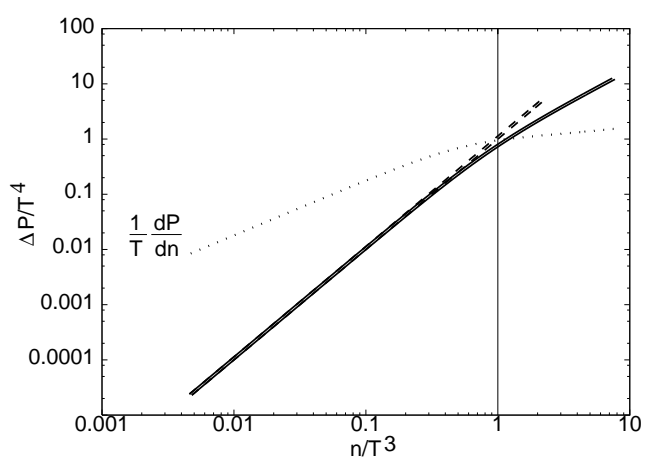

Figure 3 . The equation of state in the continuum limit at finite chemical potential for $T=2 T_{c}$ with the leading term only (dashed line), and the first two terms (full line) of the Taylor series. The band shows the 1- $\sigma$ error due to statistical errors in the susceptibilities. The EOS at $1.5 T_{c}$ and $3 T_{c}$ is indistinguishable at this scale. The dotted line is the response function $\partial\left(P / T^{4}\right) / \partial\left(n / T^{3}\right)$ at fixed $\mu$ and $T$ - the instability in $[3]$ is an artifact.

The remaining terms change the predictions by less than the statistical error band shown. Away from $T_{c}$, this justifies, post-facto, the scaling of the EOS obtained by reweighting at $N_{t}=4$ to give the continuum limit, as performed in [2]. Finally, Figure 3 shows that the measurement of susceptibilities is the simplest and most accurate route to the EOS in this range of $T$ and $\mu$ which is important for experiments.

\section{REFERENCES}

1. R. V. Gavai and S. Gupta, Phys. Rev., D1, July, 2003.

2. Z. Fodor et al., hep-lat/0208078

3. C. R. Allton et al., Phys. Rev., D68 (2003) 014507.

4. R. V. Gavai and S. Gupta, Phys. Rev., D 67 (2003) 034501.

5. T. Schäfer and F. Wilczek, Phys. Rev. Lett., 82 (1999) 3956.

6. J. Cleymans, J. Phys., G 28 (2002) 1575. 